\title{
Enhash: A Fast Streaming Algorithm For Concept Drift Detection
}

\author{
Aashi Jindal ${ }^{1}$, Prashant Gupta $^{1}$, Debarka Sengupta ${ }^{2,3}$ and Jayadeva ${ }^{1}$ \\ 1- Indian Institute of Technology Delhi - Department of Electrical Engineering \\ Hauz Khas, Delhi 110016, India \\ 2- Indraprastha Institute of Information Technology - \\ Department of Computer Science and Engineering, Delhi 110020, India \\ 3- Queensland University of Technology - Institute of Health and Biomedical Innovation \\ Brisbane, QLD 4000, Australia
}

\begin{abstract}
We propose Enhash, a fast ensemble learner that detects concept drift in a data stream. A stream may consist of abrupt, gradual, virtual, or recurring events, or a mixture of various types of drift. Enhash employs projection hash to insert an incoming sample. Benchmark tests on 6 artificial and 4 real data sets consisting of various types of drift show that Enhash is competitive with stateof-the-art ensemble learners while being significantly faster. It also has moderate resource requirements.
\end{abstract}

\section{Introduction}

A data stream environment is often characterized by large volumes of data that flow rapidly and continuously. They are processed in an online fashion to accommodate data that cannot reside in main memory. A streaming data environment is commonly used for tasks such as making recommendations for users on streaming platforms, and realtime analysis inside IoT devices. In such a stream, the underlying data distribution may change, and this phenomenon is referred to as concept drift. Formally, the posterior probability of a sample's class changes with time. Consequently, the method must also be able to adapt to the new distribution. To adapt to a new concept, the method may require supplemental or replacement learning. Tuning a model with new information is termed as supplemental learning. Replacement learning refers to the case when the model's old information becomes irrelevant, and is replaced by new information. A shift in the likelihood of observing a data point $x$ within a particular class when class boundaries are altered, is called real concept drift. Concept drift without an overlap of true class boundaries, or an incomplete representation of the actual environment, is referred to as virtual concept drift. In virtual concept drift, one requires supplemental learning, while real concept drift requires replacement learning [1]. The other common way to categorize concept drift is determined by the speed with which changes occur [2]. Hence, drift may be incremental, abrupt or gradual. A reoccurring drift is one that emerges repeatedly. Thus, in order to handle concept drift, a model must be adaptive to non-stationary environments.

Several methods have been recently proposed to handle concept drift in a streaming environment. The most popular of these are ensemble learners $[3,4,5,6,7,8]$. As the data stream evolves, an ensemble method selectively retains a few learners to maintain prior knowledge while discarding and adding new learners to learn new knowledge. 
ESANN 2021 proceedings, European Symposium on Artificial Neural Networks, Computational Intelligence and Machine Learning. Online event, 6-8 October 2021, i6doc.com publ., ISBN 978287587082-7.

Available from http://www.i6doc.com/en/.

Thus, an ensemble method is quite flexible, and maintains the stability-plasticity balance i.e. retaining the previous knowledge (stability) and learning new concepts (plasticity).

In this paper, we propose Enhash, an ensemble learner that employs projection hash to handle concept drift. For incoming samples, it generates a hash code such that similar samples tend to hash into the same bucket. A gradual forgetting factor weights the contents of a bucket. Thus, the contents of a bucket are relevant as long as the incoming stream belongs to the concept represented by them.

\section{The Proposed Method}

We propose Enhash, an ensemble learner, that employs hashing for concept drift detection. Let $x_{t} \in \mathbb{R}^{d}$ represent a sample from a data stream $S$ at time step $t$ and let $y \in\{1,2, \ldots, C\}$ represent its corresponding concept, where $C$ is the total number of concepts. Further, let us assume a family of hash functions $H$ such that $\forall h_{l} \in H$, it maps $x_{t}$ to an integer value. The hash code $h_{l}\left(x_{t}\right)$ is assigned to $x_{t}$ by hash function $h_{l}$. A bucket is a set of samples with the same hash code; both these terms are used interchangeably. The total number of samples in bucket $h_{l}\left(x_{t}\right)$ is denoted by $N_{h_{l}\left(x_{t}\right)}$. Further assume that $N$ samples have been seen and hashed from the stream so far. Amongst $N, N_{c}$ samples belong to the concept class $c$ such that $\sum_{c=1}^{c=C} N_{c}=N$. Based on the evidence from the data stream seen so far, the probability of bucket $h_{l}\left(x_{t+1}\right)$ is given by $p\left(h_{l}\left(x_{t+1}\right)\right)=\frac{N_{h_{l}\left(x_{t+1}\right)}}{N}$ and prior for class $c$ is given by $p(c)=N_{c} / N$. Assuming, $\left(N_{h_{l}\left(x_{t+1}\right)}\right)_{c}$ represents the samples of concept $c$ in bucket $h_{l}\left(x_{t+1}\right)$. Hence, the likelihood of $x_{t+1}$ belonging to concept $c$ in bucket $h_{l}\left(x_{t+1}\right)$ is given by $p\left(h_{l}\left(x_{t+1}\right) \mid c\right)=$ $\frac{\left(N_{h_{l}\left(x_{t+1}\right)}\right)_{c}}{N_{c}}$ and the probability of $x_{t+1}$ belonging to class $c$ is given by

$$
p\left(c \mid h_{l}\left(x_{t+1}\right)\right)=\frac{p\left(h_{l}\left(x_{t+1}\right) \mid c\right) p(c)}{p\left(h_{l}\left(x_{t+1}\right)\right)}=\frac{\left(N_{h_{l}\left(x_{t+1}\right)}\right) c}{N_{h_{l}\left(x_{t+1}\right)}}
$$

Equation (1) is simply the normalization of counts in bucket $h_{l}\left(x_{t+1}\right)$.

To predict the concept class of $x_{t+1}$, an ensemble of $L$ such hash functions can be employed and the weight for each concept class is computed as

$$
\hat{p}_{c}=\sum_{l=1}^{l=L} \log \left(1+p\left(c \mid h_{l}\left(x_{t+1}\right)\right)\right)
$$

and the concept class is predicted as

$$
\hat{y}=\arg \max _{c \in\{1, \ldots, C\}} \hat{p}_{c}
$$

To accommodate an incoming sample of class $c$, the bucket is updated as

$$
\left(N_{h_{i}\left(x_{t+1}\right)}\right)_{c}=1+\left(N_{h_{i}\left(x_{t+1}\right)}\right)_{c} \forall c \in\{1, . ., C\}
$$

Enhash utilizes a simple strategy as described above to build an ensemble learner for concept drift detection. 


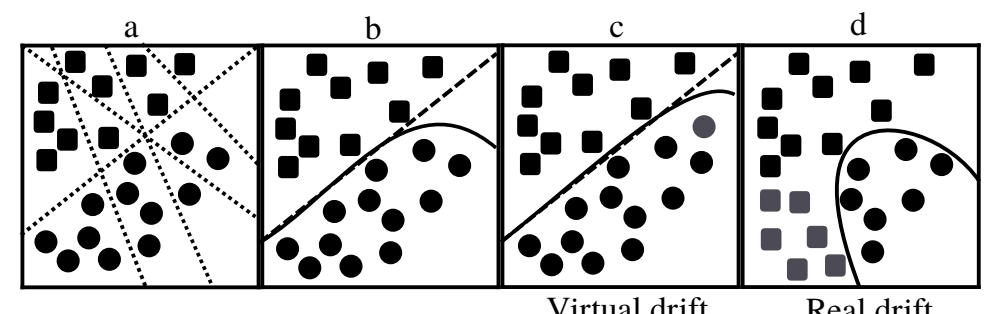

Fig. 1: Enhash accommodates both virtual and real drift.

In Enhash, the projection hash family is selected as a base learner. Here, a hash function involves the dot product of hyperplane $w^{(l)}$ and sample $x_{t}$. It is defined as

$$
h_{l}\left(x_{t}\right)=\left\lfloor\frac{1}{\text { bin-width }}\left(\sum_{j=1}^{j=d}\left(w_{j}^{(l)} *\left(x_{t}\right)_{j}\right)+\operatorname{bias}^{(l)}\right)\right\rfloor
$$

where, bin-width is quantization width, $w_{j}^{(l)}$ and bias ${ }^{(l)}$ can be sampled from any desired distribution. In our implementation, $w_{j}^{(l)} \sim N(0,1)$ and bias $^{(l)} \sim[$-bin-width, bin-width $]$.

Effectively, each $h_{l}(5)$ divides the space into equally spaced unbounded regions of size bin-width (earlier referred to as bucket). Equation (1) computes the probability of each concept class in a region. An ensemble of hash functions makes all the regions bounded. The weight of a concept class in the bounded region is computed using (2). An absolute value of concept class is assigned to each region in (3). Figure 1a shows the arrangement of randomly generated hyperplanes. The solid line in Figure $1 \mathrm{~b}$ shows the inferred decision boundary (learned distribution) after an absolute assignment of concept class to every bounded region. The dashed line in Figure $1 \mathrm{~b}$ depicts the true decision boundary (true distribution).

Assuming at time step $t$, Figure $1 \mathrm{~b}$ shows the current stage of learner. At step $t+1$, a new sample $x_{t+1}$ arrives (gray sample in Figure 1c). After updating the bucket (4), the learned distribution shifts and moves towards the true distribution. Hence, Enhash accommodates virtual drift present in the data stream.

Figure 1d depicts the real drift when the true distribution evolves. This requires forgetting some of the previously learned information. Suppose that sample $x_{t+\Delta t}$ hashes to bucket $h_{l}\left(x_{t+\Delta t}\right)$ at time say, $t+\Delta t$. Assume that previously, $x_{t}$ from a different concept, was hashed to this bucket. In order to accommodate forgetting (6), Enhash employs a decay factor multiplier to $p\left(c \mid h_{l}\left(x_{t+\Delta t}\right)\right)$ (2) while weighting a bounded region.

$$
\hat{p}_{c}=\sum_{l=1}^{l=L} \log \left(1+2^{-\lambda \Delta t} p\left(c \mid h_{l}\left(x_{t+\Delta t}\right)\right)\right)
$$

where $\lambda$ is the decay rate. The update rule for the bucket (3) is also changed to reflect 
ESANN 2021 proceedings, European Symposium on Artificial Neural Networks, Computational Intelligence and Machine Learning. Online event, 6-8 October 2021, i6doc.com publ., ISBN 978287587082-7.

Available from http://www.i6doc.com/en/.

the new concept class as $\left(N_{h_{l}\left(x_{t+1}\right)}\right)_{c}=1+2^{-\lambda \Delta t}\left(N_{h_{l}\left(x_{t+1}\right)}\right)_{c}$. Setting $\lambda=0$ will reduce these equations to the base case.

In order to break ties in $p\left(c \mid h_{l}\left(x_{t}\right)\right)$, the class weight in the region is also weighted by the distance of the sample $x_{t}$ from the mean of class samples in bucket $h_{l}\left(x_{t}\right)$, i.e. $\operatorname{mean}_{h_{l}\left(x_{t}\right)}^{c}$

$$
\begin{gathered}
\operatorname{dist}_{c}\left(x_{t}\right)=\sqrt{\sum_{i=1}^{i=d}\left(\left(x_{t}\right)_{i}-\left(\operatorname{mean}_{h_{l}\left(x_{t}\right)}^{c}\right)_{i}\right)^{2}} \\
\left.\hat{p}_{c}=\sum_{l=1}^{l=L} \log \left(1+\frac{2^{-\lambda \Delta t} p\left(c \mid h_{l}\left(x_{t}\right)\right)}{\operatorname{dist}_{c}\left(x_{t}\right)}\right)\right)
\end{gathered}
$$

and remaining ties are broken arbitrarily. Here, $\left(x_{t}\right)_{i}$ denotes the $i$-th feature of sample $x_{t}$.

\section{Experimental Setup}

Enhash's performance was compared with some of the widely used ensemble learners. These include Learn ${ }^{++}$.NSE [3], Accuracy-Weighted Ensemble (AWE) [7], Additive Expert Ensemble (AEE) [8], Online Bagging-ADWIN (OB) [9], Leveraging Bagging (LB) [5], Online SMOTE Bagging (OSMOTEB) [4], ARF [6], Online CSB2 [4], Online RUSBoost [4], and Streaming Random Patches (SRP) [10]. The implementation of these methods is available in scikit-multiflow python package [11]. A fixed value of estimators was used for all the methods. For methods AWE, Learn ${ }^{++}$.NSE, $\mathrm{LB}$, and $\mathrm{OB}$, the maximum size of window was set $\min (5000,0.1 * n)$, where $n$ is the total number of samples. For the rest of the parameters, the default value was used for all the methods.

\section{Experimental Results}

For all methods, the number of estimators is considered as 10. In addition, for Enhash the bin-width was set to $\{0.1,0.01\}$ and $\lambda$ was set to 0.015 . Table 1 compares the performances in terms of error and KappaM using Interleaved Test-Train strategy. For these measures, the performance of the proposed method was superior to AWE, AEE, and Online RUSBoost on 8 data sets, Online CSB2, Learn ${ }^{++}$.NSE, OSMOTEB, and OB on 7 data sets, Learn++ and LB on 6 data sets, ARF on 5 data sets, and SRP on 4 data sets. The performance of Enhash supersedes all other methods for 3 data sets.

Other evaluation criteria are speed and RAM-hours (Table 2). For all data sets, Enhash takes the least time. In terms of speed, Learn ${ }^{++}$.NSE is the closest competitor of Enhash. In terms of accuracy, however, Enhash supersedes Learn ${ }^{++}$.NSE on the majority of the data sets. Our findings were also consistent for error and KappaM. The overall closest competitors of Enhash in terms of error and KappaM are SRP, ARF, LB, and OB. Enhash's speed and RAM-hours' requirement are almost insignificant when 
ESANN 2021 proceedings, European Symposium on Artificial Neural Networks, Computational Intelligence and Machine Learning. Online event, 6-8 October 2021, i6doc.com publ., ISBN 978287587082-7.

Available from http://www.i6doc.com/en/.

\begin{tabular}{|c|c|c|c|c|c|c|c|c|c|c|c|}
\hline Dataset & $\begin{array}{r}\text { Learn }^{++} \\
. \text {NSE }\end{array}$ & LB & OB & $\begin{array}{r}\text { OSMO- } \\
\text {-TEB }\end{array}$ & AWE & AEE & $\mathrm{ARF}$ & Enhash & $\begin{array}{r}\text { Online } \\
\text { CSB2 }\end{array}$ & $\begin{array}{r}\text { Online } \\
\text { RUSBoost }\end{array}$ & SRP \\
\hline \multicolumn{12}{|l|}{ (a) Error (in \%) } \\
\hline transientChessboard & 3.67 & 13.13 & 29.62 & 24.28 & 89.78 & 85.37 & 27.56 & 18.84 & 19.80 & 23.95 & 39.53 \\
\hline rotatingHyperplane & 24.13 & 24.65 & 15.08 & 19.75 & 16.27 & 18.10 & 16.40 & 32.72 & 18.16 & 28.06 & 17.69 \\
\hline mixedDrift & 39.46 & 16.71 & 23.63 & 20.30 & 77.79 & 81.17 & 19.79 & 12.88 & 18.78 & 16.80 & 19.69 \\
\hline movingSquares & 68.74 & 55.69 & 65.42 & 61.12 & 67.51 & 67.23 & 58.54 & 13.29 & 64.43 & 35.34 & 19.40 \\
\hline interchangingRBF & 75.43 & 4.61 & 37.67 & 22.05 & 83.49 & 82.58 & 3.22 & 2.72 & 3.66 & 97.37 & 2.69 \\
\hline interRBF20D & 76.36 & 5.62 & 37.02 & 21.00 & 84.90 & 82.35 & 2.39 & 11.30 & 3.48 & 95.73 & 2.92 \\
\hline airlines & 42.92 & 43.36 & 39.23 & 40.66 & 38.49 & 39.17 & 33.09 & 41.66 & 43.04 & 44.41 & 32.62 \\
\hline elec2 & 34.63 & 18.64 & 23.26 & 24.60 & 39.64 & 26.71 & 11.59 & 17.34 & 22.94 & 18.95 & 11.81 \\
\hline NEweather & 29.20 & 27.20 & 20.84 & 22.74 & 30.72 & 30.78 & 21.43 & 29.27 & 23.27 & 29.20 & 21.23 \\
\hline outdoorStream & - & 9.33 & 34.15 & 21.25 & 78.55 & 42.45 & 26.12 & 8.75 & 33.85 & 62.73 & 34.62 \\
\hline \multicolumn{12}{|l|}{ (b) KappaM } \\
\hline transientChessboard & 0.96 & 0.85 & 0.66 & 0.72 & -0.03 & 0.02 & 0.68 & 0.78 & 0.77 & 0.73 & 0.55 \\
\hline rotatingHyperplane & 0.52 & 0.51 & 0.70 & 0.60 & 0.67 & 0.64 & 0.67 & 0.34 & 0.64 & 0.44 & 0.65 \\
\hline mixedDrift & 0.54 & 0.80 & 0.72 & 0.76 & 0.09 & 0.05 & 0.77 & 0.85 & 0.78 & 0.80 & 0.77 \\
\hline movingSquares & 0.08 & 0.26 & 0.13 & 0.19 & 0.10 & 0.10 & 0.22 & 0.82 & 0.14 & 0.53 & 0.74 \\
\hline interchangingRBF & 0.18 & 0.95 & 0.59 & 0.76 & 0.09 & 0.10 & 0.96 & 0.97 & 0.96 & -0.06 & 0.97 \\
\hline interRBF20D & 0.17 & 0.94 & 0.60 & 0.77 & 0.08 & 0.10 & 0.97 & 0.88 & 0.96 & -0.04 & 0.97 \\
\hline airlines & 0.04 & 0.03 & 0.12 & 0.09 & 0.14 & 0.12 & 0.26 & 0.06 & 0.03 & 0.00 & 0.27 \\
\hline elec2 & 0.18 & 0.56 & 0.45 & 0.42 & 0.07 & 0.37 & 0.73 & 0.59 & 0.46 & 0.55 & 0.72 \\
\hline NEweather & 0.07 & 0.13 & 0.34 & 0.28 & 0.02 & 0.02 & 0.32 & 0.07 & 0.26 & 0.07 & 0.32 \\
\hline outdoorStream & - & 0.90 & 0.65 & 0.78 & 0.19 & 0.56 & 0.73 & 0.91 & 0.65 & 0.36 & 0.64 \\
\hline
\end{tabular}

Table 1: For a given data set, the method with the best metric value is in boldface. Due to implementation constraint, Learn ${ }^{++}$.NSE could not run for the outdoorStream data set.

\begin{tabular}{|c|c|c|c|c|c|c|c|c|c|c|c|}
\hline Dataset & $\begin{array}{r}\text { Learn }^{++} \\
. \text {NSE }\end{array}$ & LB & OB & $\begin{array}{r}\text { OSMO- } \\
\text {-TEB }\end{array}$ & AWE & AEE & ARF & Enhash & $\begin{array}{r}\text { Online } \\
\text { CSB2 }\end{array}$ & $\begin{array}{r}\text { Online } \\
\text { RUSBoost }\end{array}$ & SRP \\
\hline \multicolumn{12}{|l|}{ Time (in hrs) } \\
\hline transientChessboard & 0.287 & 8.374 & 0.983 & 13.623 & 0.181 & 0.570 & 0.481 & 0.099 & 1.081 & 1.008 & 1.238 \\
\hline rotatingHyperplane & 0.199 & 14.022 & 6.957 & 114.615 & 0.198 & 0.660 & 1.318 & 0.067 & 2.917 & 4.109 & 1.635 \\
\hline mixedDrift & 0.627 & 27.408 & 7.916 & 185.501 & 1.673 & 17.149 & 1.875 & 0.339 & 5.708 & 4.428 & 5.456 \\
\hline movingSquares & 0.179 & 9.015 & 4.180 & 28.028 & 0.142 & 0.398 & 7.082 & 0.068 & 1.641 & 2.202 & 0.812 \\
\hline interchangingRBF & 0.185 & 8.298 & 1.106 & 7.424 & 0.376 & 1.048 & 0.411 & 0.132 & 1.411 & 1.346 & 2.005 \\
\hline interRBF20D & 0.166 & 19.167 & 1.292 & 7.604 & 2.091 & 2.373 & 2.940 & 0.106 & 1.757 & 1.447 & 8.569 \\
\hline airlines & 0.571 & 35.839 & 13.088 & 403.378 & 0.744 & 4.357 & 2.40 & 0.182 & 6.553 & 8.906 & 3.388 \\
\hline elec2 & 0.020 & 7.575 & 1.777 & 14.511 & 0.016 & 0.059 & 0.171 & 0.015 & 0.585 & 0.871 & 0.306 \\
\hline NEweather & 0.008 & 0.590 & 0.183 & 1.326 & 0.013 & 0.020 & 0.080 & 0.006 & 0.219 & 0.282 & 0.140 \\
\hline outdoorStream & - & 0.069 & 0.093 & 0.162 & 0.047 & 0.129 & 0.067 & 0.004 & 0.191 & 0.182 & 0.293 \\
\hline \multicolumn{12}{|l|}{ RAM-hours } \\
\hline transientChesst & $8.1 \mathrm{e}-05$ & $8.0 \mathrm{e}-02$ & $3.3 \mathrm{e}-04$ & $5.5 \mathrm{e}-01$ & $2.3 \mathrm{e}-04$ & $5.5 \mathrm{e}-05$ & $1.2 \mathrm{e}-03$ & $4.4 \mathrm{e}-04$ & $7.5 \mathrm{e}-04$ & $7.7 \mathrm{e}-04$ & $3.3 \mathrm{e}-04$ \\
\hline rotatingHyper & $5.6 \mathrm{e}-05$ & $3.5 \mathrm{e}-01$ & $1.7 \mathrm{e}-01$ & $4.6 \mathrm{e}+01$ & $5.8 \mathrm{e}-04$ & $1.1 \mathrm{e}-04$ & $3.4 \mathrm{e}-02$ & $7.8 \mathrm{e}-05$ & $1.7 \mathrm{e}-02$ & $2.4 \mathrm{e}-02$ & $1.2 \mathrm{e}-01$ \\
\hline mixedDrift & $4.9 \mathrm{e}-04$ & $2.7 \mathrm{e}-01$ & $2.9 \mathrm{e}-02$ & $3.9 \mathrm{e}+01$ & $2.5 \mathrm{e}-03$ & $2.9 \mathrm{e}-03$ & $1.3 \mathrm{e}-02$ & $5.3 \mathrm{e}-03$ & $1.3 \mathrm{e}-02$ & $2.1 \mathrm{e}-03$ & $2.3 \mathrm{e}-03$ \\
\hline movingSquares & $4.9 \mathrm{e}-05$ & $8.7 \mathrm{e}-02$ & $3.9 \mathrm{e}-02$ & $2.3 e+00$ & $1.6 \mathrm{e}-04$ & $2.3 \mathrm{e}-05$ & $3.0 \mathrm{e}+00$ & $1.2 \mathrm{e}-05$ & $4.8 \mathrm{e}-03$ & $6.4 \mathrm{e}-03$ & $1.4 \mathrm{e}-03$ \\
\hline interchangingRBF & $5.2 \mathrm{e}-05$ & $8.2 \mathrm{e}-02$ & $6.1 \mathrm{e}-04$ & $2.4 \mathrm{e}-01$ & $5.6 \mathrm{e}-04$ & $1.8 \mathrm{e}-04$ & $7.6 e-04$ & $9.9 \mathrm{e}-05$ & $1.1 \mathrm{e}-03$ & $6.1 \mathrm{e}-04$ & $6.9 \mathrm{e}-04$ \\
\hline interRBF20D & $1.7 \mathrm{e}-04$ & $7.8 \mathrm{e}-01$ & $2.4 \mathrm{e}-03$ & $1.1 \mathrm{e}+00$ & $1.8 \mathrm{e}-02$ & $3.3 \mathrm{e}-03$ & $2.5 \mathrm{e}-03$ & $1.0 \mathrm{e}-03$ & $3.5 \mathrm{e}-03$ & $1.5 \mathrm{e}-03$ & $5.8 \mathrm{e}-01$ \\
\hline airlines & $1.3 \mathrm{e}-03$ & $6.9 \mathrm{e}-01$ & $2.4 \mathrm{e}-01$ & $3.0 \mathrm{e}+02$ & $1.7 \mathrm{e}-03$ & $5.2 \mathrm{e}-04$ & $9.3 \mathrm{e}-02$ & $1.6 \mathrm{e}-01$ & $3.1 \mathrm{e}-02$ & $4.2 \mathrm{e}-02$ & $1.7 \mathrm{e}-01$ \\
\hline elec2 & $3.7 \mathrm{e}-06$ & $1.4 \mathrm{e}-01$ & $3.3 e-02$ & $1.3 \mathrm{e}+00$ & $3.2 \mathrm{e}-05$ & $8.0 \mathrm{e}-06$ & $1.4 \mathrm{e}-03$ & $1.8 \mathrm{e}-05$ & $2.7 \mathrm{e}-03$ & $4.4 \mathrm{e}-03$ & $5.5 \mathrm{e}-03$ \\
\hline NEweather & $7.0 \mathrm{e}-07$ & $4.6 \mathrm{e}-03$ & $1.4 \mathrm{e}-03$ & $3.1 \mathrm{e}-02$ & $1.2 \mathrm{e}-05$ & $2.6 \mathrm{e}-06$ & $1.2 \mathrm{e}-03$ & $1.7 \mathrm{e}-04$ & $1.1 \mathrm{e}-03$ & $1.3 \mathrm{e}-03$ & $4.5 \mathrm{e}-03$ \\
\hline outdoorStream & - & $2.6 \mathrm{e}-04$ & $1.4 \mathrm{e}-03$ & $6.0 \mathrm{e}-03$ & $1.4 \mathrm{e}-04$ & $4.5 \mathrm{e}-04$ & $9.4 \mathrm{e}-05$ & $3.4 \mathrm{e}-05$ & $5.8 \mathrm{e}-03$ & $3.2 \mathrm{e}-03$ & $6.5 \mathrm{e}-04$ \\
\hline
\end{tabular}

Table 2: (a) The method with the fastest speed is highlighted for every data set. (b) The memory consumption is measured in terms of RAM-hours. The method with the least value of RAM-hours is highlighted for every data set. 
ESANN 2021 proceedings, European Symposium on Artificial Neural Networks, Computational Intelligence and Machine Learning. Online event, 6-8 October 2021, i6doc.com publ., ISBN 978287587082-7.

Available from http://www.i6doc.com/en/.

compared with other methods. OSMOTEB is the slowest when compared with all other methods. The remaining methods AWE and AEE have inadequate performances as compared to Enhash.

In summary, LB and ARF suffer in detecting incremental drifts. However, LB and ARF have an overall satisfactory performance. Enhash performs relatively well on all data sets. Enhash has a superior performance on a data set consisting of three different kinds of drifts, namely incremental, virtual, and abrupt drifts.

\section{Conclusions}

We conclude that Enhash supersedes other methods in terms of speed since the algorithm effectively requires only $\mathcal{O}(1)$ running time for each sample on a given estimator. In addition, the performance of Enhash in terms of error and KappaM is better or comparable to others for majority data sets. These data sets constitute abrupt, gradual, virtual, and reoccurring drift phenomena. The closest competitor of Enhash in terms of performance is ARF and SRP. Notably, Enhash requires, on an average 10 times lesser RAM-hours than that of ARF and SRP.

\section{References}

[1] João Gama, Indrè Žliobaitė, Albert Bifet, Mykola Pechenizkiy, and Abdelhamid Bouchachia. A survey on concept drift adaptation. ACM computing surveys (CSUR), 46(4):44, 2014.

[2] Roberto Souto Maior de Barros and Silas Garrido T de Carvalho Santos. An overview and comprehensive comparison of ensembles for concept drift. Information Fusion, 52:213-244, 2019.

[3] Ryan Elwell and Robi Polikar. Incremental learning of concept drift in nonstationary environments IEEE Transactions on Neural Networks, 22(10):1517-1531, 2011.

[4] Boyu Wang and Joelle Pineau. Online bagging and boosting for imbalanced data streams. IEEE Transactions on Knowledge and Data Engineering, 28(12):3353-3366, 2016.

[5] Albert Bifet, Geoff Holmes, and Bernhard Pfahringer. Leveraging bagging for evolving data streams. In Joint European conference on machine learning and knowledge discovery in databases, pages 135-150. Springer, 2010.

[6] Heitor M Gomes, Albert Bifet, Jesse Read, Jean Paul Barddal, Fabrício Enembreck, Bernhard Pfharinger, Geoff Holmes, and Talel Abdessalem. Adaptive random forests for evolving data stream classification. Machine Learning, 106(9-10):1469-1495, 2017.

[7] Haixun Wang, Wei Fan, Philip S Yu, and Jiawei Han. Mining concept-drifting data streams using ensemble classifiers. In Proceedings of the ninth ACM SIGKDD international conference on Knowledge discovery and data mining, pages 226-235. AcM, 2003.

[8] Jeremy Z Kolter and Marcus A Maloof. Using additive expert ensembles to cope with concept drift. In Proceedings of the 22nd international conference on Machine learning, pages 449-456. ACM, 2005.

[9] Nikunj C Oza. Online bagging and boosting. In 2005 IEEE international conference on systems, man and cybernetics, volume 3, pages 2340-2345. Ieee, 2005.

[10] Heitor Murilo Gomes, Jesse Read, and Albert Bifet. Streaming random patches for evolving data stream classification. In 2019 IEEE International Conference on Data Mining (ICDM), pages 240-249. IEEE, 2019.

[11] Jacob Montiel, Jesse Read, Albert Bifet, and Talel Abdessalem. Scikit-multiflow: A multi-output streaming framework. Journal of Machine Learning Research, 19(72):1-5, 2018. 